\title{
Transplantation of haemochromatosis liver and intestine into a normal recipient
}

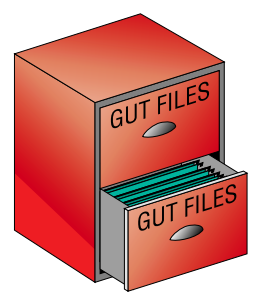

Background-Haemochromatosis is a common genetic disease leading to iron overload. Although the gene had been identified (HFE), ${ }^{1}$ the pathogenesis had not been fully elucidated. ${ }^{2}$ The inadvertent transplant of a C282Y homozygous liver and intestine provided a unique opportunity to study this problem.

Methods-A 19 year old man underwent orthotopic liver and intestinal transplantation in January 1997 for the treatment of short bowel syndrome secondary to a mid gut volvulus with resection and cholestatic liver disease resulting from total parenteral nutrition. The organ donor was an 18 year old woman posthumously discovered to be a C282Y homozygote for haemochromatosis.

Results-Preoperative recipient blood tests included a serum ferritin of $34 \mu \mathrm{g} / 1$ (normal range 15-300 $\mu \mathrm{g} / \mathrm{l}$ ) and a transferrin saturation of $10 \%$ (normal 20-55\%). Transplantation of the intestine and liver was performed as previously described at this medical centre and included duodenum, jejunum, and ileum. ${ }^{3}$ At 21 months after transplantation the recipient had a great increase in transferrin saturation at $94 \%$ with a normal serum ferritin of $103 \mu \mathrm{g} / 1$. Hepatic iron concentration at four months was 20 $\mu \mathrm{mol} / \mathrm{g}$ and at $22 \mathrm{months}$ was $22.3 \mu \mathrm{mol} / \mathrm{g}$ (normal 0-35 $\mu \mathrm{mol} / \mathrm{g}$ ). Genetic testing for haemochromatosis ${ }^{4}$ on his liver graft revealed homozygosity for the C282Y mutation of the $H F E$ gene. Genetic testing on a peripheral blood sample at 21 months was normal (wild type) for the C282Y mutation. The donor family was investigated for haemochromatosis (fig 1). A brother of the donor was an iron loaded homozygote and her mother appeared to be a nonexpressing homozygote. The mother had a non-identical

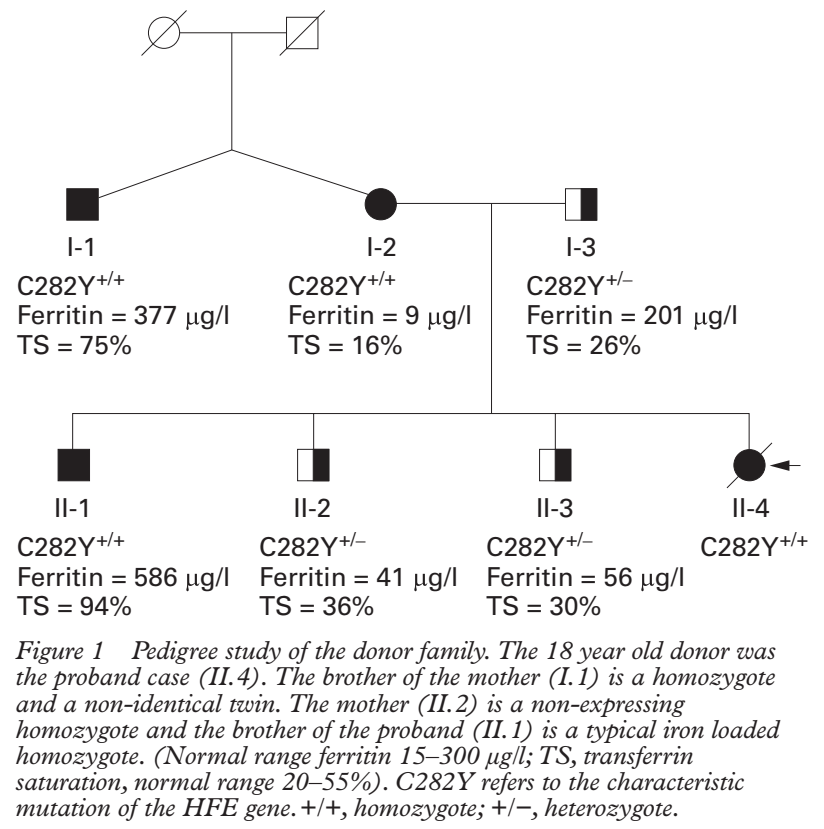

twin that was homozygous for the C282Y mutation with abnormal iron studies.

Conclusions - This case suggests that the genetic defect of haemochromatosis has been transplanted into the recipient with the donor intestine and that iron accumulation will probably occur with time. Within 21 months of transplantation the recipient is showing evidence of the typical biochemical abnormality seen in a young patient with haemochromatosis, namely an increase in transferrin saturation with a normal hepatic iron concentration. Although the serum ferritin is normal, it is likely that if untreated it will continue to rise with time. Therefore, we have identified a predisposition to future iron overload rather than iron overload at 21 months. The concomitant transplantation of the haemochromatosis liver is less likely to be contributing to the abnormal iron metabolism. Transplantation of a haemochromatosis liver alone into a normal recipient has been previously documented at this centre with a progressive decline in hepatic iron concentration and a normal radioiron absorption study. ${ }^{5}$ This supports the hypothesis that the fundamental defect in haemochromatosis is site specific at the level of the intestine rather than a systemic abnormality.

C ADAMS

G JEFFREY

Department of Medicine,

University of Western Ontario, London, Ontario, Canada

K ALANEN

S CHAKRABARTI

Department of Pathology,

University of Western Ontario,

London, Ontario, Canada

R PRESHAW

Department of Surgery,

University of Calgary,

Calgary, Alberta, Canada

W HOWSON

Department of Laboratory Medicine,

University of Western Ontario,

London, Ontario, Canada

D GRANT

Department of Surgery,

University of Western Ontario,

London, Ontario, Canada

Correspondence to: Paul C Adams, MD, Department of Medicine, London Health Sciences Centre, 339 Windermere Road, London, Ontario, Canada N6A 5A5. Email: padams@julian.uwo.ca

1 Feder JN, Gnirke A, Thomas W, et al. A novel MHC class I-like gene is mutated in patients with hereditary hemochromatosis. Nat Genet 1996;13:399-408

2 Bacon BR, Powell L, Adams PC, et al. Molecular medicine and hemochromatosis: at the crossroads. Gastroenterology 1999;116:193-207. 3 Grant D, Wall W, Mimeault R, et al. Successful small bowel-liver transplantation. Lancet 1990;335:181-4.

4 Adams PC, Chakrabarti S. Genotypic/phenotypic correlations in genetic hemochromatosis: evolution of diagnostic criteria. Gastroenterology 1998; 114:319-23.

5 Adams PC, Ghent CN, Grant DR, et al. Transplantation of haemochromatosis liver into donor: evidence against an inherited intrahepatic defect. Gut 1991;32:1082-3. 